\title{
Maximum Principle for Forward-Backward Stochastic Control System Driven by Lévy Process
}

\author{
Xiangrong Wang ${ }^{1}$ and Hong Huang ${ }^{2}$ \\ ${ }^{1}$ College of Mathematics and Systems Science, Shandong University of Science and Technology, Qingdao 266590, China \\ ${ }^{2}$ College of Information Science and Engineering, Shandong University of Science and Technology, Qingdao 266590, China \\ Correspondence should be addressed to Hong Huang; huangqian1201@163.com
}

Received 4 February 2015; Revised 26 April 2015; Accepted 12 May 2015

Academic Editor: Son Nguyen

Copyright (c) $2015 \mathrm{X}$. Wang and H. Huang. This is an open access article distributed under the Creative Commons Attribution License, which permits unrestricted use, distribution, and reproduction in any medium, provided the original work is properly cited.

We study a stochastic optimal control problem where the controlled system is described by a forward-backward stochastic differential equation driven by Lévy process. In order to get our main result of this paper, the maximum principle, we prove the continuity result depending on parameters about fully coupled forward-backward stochastic differential equations driven by Lévy process. Under some additional convexity conditions, the maximum principle is also proved to be sufficient. Finally, the result is applied to the linear quadratic problem.

\section{Introduction}

The stochastic optimal control problem is one of the central themes of modern control science. Forward-backward stochastic control systems which the controlled systems described by forward-backward stochastic differential equations (FBSDEs) are widely used in mathematics and finance. Peng and $\mathrm{Wu}$ [1] firstly used a probabilistic method to get the existence and uniqueness results of fully coupled FBSDEs; then Peng [2] considered one kind of forward-backward stochastic control systems with economic background when the control domain is convex and obtained the maximum principle; since then a number of developments in this direction were reported in $\mathrm{Wu}$ [3] and Shi and $\mathrm{Wu}$ [4]. $\mathrm{Wu}$ [5] firstly proved the existence and uniqueness results of the solutions to fully coupled FBSDEs with Brownian motion and Poisson process; then Shi and $\mathrm{Wu}$ [6] got the stochastic maximum principle for fully coupled FBSDEs with random jumps. More conclusions about stochastic maximum principle about forward-backward stochastic control systems driven by Brownian motion and Poisson process can be seen in [7-9].

It is natural to extend the stochastic differential equations (SDEs) with Brownian motion and Poisson process to the case of Lévy process with independent and stationary increments. Baghery et al. [10] firstly considered the following fully coupled forward-backward stochastic differential equation driven by Lévy process (FBSDEL):

$$
\begin{aligned}
d x_{t}= & b\left(t, x_{t}, y_{t}, z_{t}, r_{t}\right) d t+\sum_{i=1}^{d} \sigma^{i}\left(t, x_{t}, y_{t}, z_{t}, r_{t}\right) d B_{t}^{i} \\
& +\sum_{i=1}^{\infty} g^{i}\left(t, x_{t-}, y_{t-}, z_{t}, r_{t}\right) d H_{t}^{i}, \\
-d y_{t}= & f\left(t, x_{t}, y_{t}, z_{t}, r_{t}\right) d t-\sum_{i=1}^{d} z_{t}^{i} d B_{t}^{i}-\sum_{i=1}^{\infty} r_{t}^{i} d H_{t}^{i}, \\
x_{0}= & a \\
y_{T}= & \Phi\left(x_{T}\right),
\end{aligned}
$$

and, under some monotonicity assumptions, they got the existence and uniqueness of solutions for this equation. Zhu [11] had proposed the asymptotic stability in the Pth moment for SDE with Lévy noise. Nualart and Schoutens [12] constructed a set of pairwise strongly orthonormal martingales called Teugels martingale and they also proved a martingale representation theorem for Lévy processes satisfying some 
exponential moment condition. Using the martingale representation theorem they [13] had proved the existence and uniqueness of a solution for backward stochastic differential equations (BSDEs) driven by Teugels martingale. Bahlali et al. [14] extended this conclusion to the BSDEs driven by Teugels martingale and an independent Brownian motion; they got the existence, uniqueness, and comparison of solutions for these equations under Lipschitz and locally Lipschitz conditions on the coefficient. Based on these consequences, Mitsui and Tabata [15] established the closeness property of the solution of the multidimensional backward stochastic Riccati differential equation with Lévy process; then they used this solution to study a linear quadratic regulation problem with Lévy process. After the foundation of the existence and uniqueness of the solutions of SDEs and multidimensional BSDEs driven by Lévy process, Tang and Wu [16] proceed to study a stochastic linear quadratic optimal control problem with a Lévy process, where the cost weighting matrices of the state and control were allowed to be indefinite.

These consequences are important for the researching of maximum principle for forward stochastic control system driven by Lévy process, as the adjoint equation for forward stochastic control system is a BSDE. Meng and Tang [17] firstly were concerned with optimal control for forward stochastic control system driven by Teugels martingale; they got the maximum principle and verification theorem for this system. In 2012, Tang and Zhang [18] were concerned with optimal control of BSDE driven by Teugels martingale and an independent multidimensional Brownian motion; they derived the necessary and sufficient conditions for the existence of the optimal control by means of convex variation methods and duality techniques. When the control domain was nonconcave and the control variable was allowed to enter the coefficients of the Teugels martingales, Lin [19] got the necessary maximum principle for optimal control of stochastic system driven by multidimensional Teugels martingales. Zhang et al. [20] firstly studied the forwardbackward stochastic control system where the system was driven by Teugels martingale and an independent multidimensional Brownian motion as follows:

$$
\begin{aligned}
d x_{t}= & b\left(t, x_{t}, u_{t}\right) d t+\sum_{i=1}^{d} \sigma^{i}\left(t, x_{t}, u_{t}\right) d B_{t}^{i} \\
& +\sum_{i=1}^{\infty} g^{i}\left(t, x_{t-}, u_{t}\right) d H_{t}^{i}, \\
-d y_{t}= & f\left(t, x_{t}, y_{t}, z_{t}, r_{t}, u_{t}\right) d t-\sum_{i=1}^{d} z_{t}^{i} d B_{t}^{i}-\sum_{i=1}^{\infty} r_{t}^{i} d H_{t}^{i}, \\
x_{0}= & a, \\
y_{T}= & \Phi\left(x_{T}\right),
\end{aligned}
$$

and they had got the maximum principle and verification theorem in the condition of the SDE part did not contain the backward state variables; the forward-backward stochastic control system they studied was not fully coupled.
In this paper, we extend the result of Zhang et al. [20] to the fully coupled forward-backward stochastic control system. Here the state variables are described by fully coupled FBSDEs driven by Brownian motion and an independent Teugels martingale. Before applying the convex variation and duality technique to obtain the stochastic maximum principle, we use the same method in [5] to get the continuity result depending on parameters, as the continuity result is not only important for us to get the stochastic maximum principle but also important property of FBSDEL especially in practice. Different from the $\mathrm{Wu}$ [3] and Shi and $\mathrm{Wu}$ [6] about maximum principles to Brownian motions and Poisson process, we also need more general Ito's formula about càdlàg semimartingale.

This paper is organized as follows. In Section 2, we will give some preliminaries used in this paper. Section 3 presents the continuity result depending on parameters about fully coupled FBSDEs driven by Lévy process. In Section 4, we obtain the main result of this paper, the maximum principle. We also prove that, under some additional convexity conditions, the maximum principle can be a sufficient condition for optimal control. And, in Section 5, an application of our stochastic maximum principle to the linear quadratic problem which the linear control system described by fully coupled FBSDEL is proved.

\section{Preliminaries and Notations}

Let $\left(\Omega, F, P, \mathscr{F}_{t}, B_{t}, L_{t}\right)(t \in[0, T])$ be a complete space driving by Brownian motion and Lévy process in $R^{m} \times R \backslash\{0\}$, with Lévy measure $v$; that is, $\left\{B_{t}\right\}_{0 \leq t \leq T}$ is a standard Brownian motion. $\left\{L_{t}\right\}_{0 \leq t \leq T}$ is $R$-valued Lévy process of the form $L_{t}=$ $b t+\ell_{t}$ independent of $\left\{B_{t}\right\}_{0 \leq t \leq T}$, corresponding to a standard Lévy measure $v$ satisfying the following conditions:

(i) $\int_{R}\left(1 \wedge x^{2}\right) \nu(d x)<\infty$,

(ii) $\int_{(-\varepsilon, \varepsilon)^{c}} e^{\lambda|x|} \nu(d x)<\infty$, for every $\varepsilon>0$ and for some $\lambda>0$, and

$$
\mathscr{F}_{t}=\sigma\left(L_{s}, s \leq t\right) \vee \sigma\left(B_{s}, s \leq t\right) \vee \aleph .
$$

Here $\aleph$ is the totality of $P$-null sets and $g_{1} \vee g_{2}$ denotes the $\sigma$-field generated by $g_{1} \cup g_{2}$.

Let $x$ be a Lévy process and denote the left limit process by $x_{t-}=\lim _{s \rightarrow t, s \leq t} x_{s}$ and the jump size at time $t$ by $\Delta x_{t}=x_{t}-x_{t-}$. Set

$$
x_{t}^{i}= \begin{cases}\sum_{0<s \leq t}\left(\Delta x_{s}\right)^{i}, & i \geq 2 \\ x_{t}, & i=1\end{cases}
$$

and we denote the compensated power jump process of order $i$ by $Y_{t}^{i}=x_{t}^{i}-E\left[x_{t}^{i}\right]$; then Teugels martingale $\left(H_{t}^{i}\right)_{0 \leq t \leq T}$ can be defined as follows:

$$
H_{t}^{i}=c_{i, i} Y_{t}^{i}+c_{i, i-1} Y_{t}^{i-1}+c_{i, i-2} Y_{t}^{i-2}+\cdots+c_{i, 1} Y_{t}^{1} .
$$

Here the coefficients $c_{i, k}$ correspond to orthonormalization of the polynomials $1, x, x^{2}, \ldots$ with respect to the measure $\mu(d x)=v(d x)+\sigma^{2} \delta_{0}(d x)$. 
Now we introduce some notations adopted in this paper as follows:

(1) H: Hilbert space,

(2) $\langle\alpha, \beta\rangle$ : the inner product in $R^{n}, \forall \alpha, \beta \in R^{n}$,

(3) $|\alpha|=\sqrt{\langle\alpha, \alpha\rangle}$ : the norm in $R^{n}, \forall \alpha \in R^{n}$,

(4) $\langle A, B\rangle=\operatorname{tr}\left(A B^{T}\right)$ : the inner product in $R^{n \times m}, \forall A, B \in$ $R^{n \times m}$

(5) $|A|=\sqrt{\operatorname{tr}\left(A A^{T}\right)}$ : the inner product in $R^{n \times m}, \forall A \in$ $R^{n \times m}$

(6) $l^{2}$ : the space of real valued sequences $X=\left(x_{n}\right)_{n \geq 0}$ such that

$$
\|x\|_{l^{2}}^{2}=\sum_{i=1}^{\infty} x_{i}^{2}<\infty
$$

(7) $l^{2}(H)$ : the space of $H$-valued sequences $\phi=\varphi^{i}{ }_{i \geq 1}$ such that

$$
\|\phi\|_{l^{2}(H)}^{2}=\sum_{i=1}^{\infty}\left\|\phi^{i}\right\|^{2}<\infty
$$

(8) $l^{2}(0, T ; H)$ : the corresponding spaces of $l^{2}(H)$ valued $\mathscr{F}_{t}$-measurable processes equipped with the norm

$$
\|\phi\|_{l^{2}(0, T ; H)}^{2}=E \int_{0}^{T} \sum_{i=1}^{\infty}\left\|\phi_{t}^{i}\right\|_{H}^{2} d t<\infty,
$$

(9) $L^{2}\left(\Omega, \mathscr{F}_{t}, P ; H\right)$ : the space of $H$-valued random variable $\xi$ with the norm

$$
\|\xi\|^{2}=E\|\xi\|_{L^{2}\left(\Omega, \mathscr{F}_{t}, P ; H\right)}^{2}<\infty
$$

(10) $M^{2}(0, T ; H)$ : the space of $H$-valued $\mathscr{F}_{t}$-measurable process $\phi(\cdot)=\{\phi(t, \omega):(t, \omega) \in[0, T] \times \Omega\}$ with the norm

$$
\|\phi(\cdot)\|_{M^{2}(0, T ; H)}^{2}=E \int_{0}^{T}\left\|\phi_{t}\right\|_{M^{2}(0, T ; H)}^{2} d t<\infty,
$$

(11) $S^{2}(0, T ; H)$ : the space of $H$-valued $\mathscr{F}_{t}$-measurable càdlàg process $f(\cdot)=\{f(t, \omega):(t, \omega) \in[0, T] \times \Omega\}$ with the norm

$$
\|f(\cdot)\|_{S^{2}(0, T ; H)}^{2}=E \sup _{0 \leq t \leq T}\left\|f_{t}\right\|_{H}^{2} d t<\infty,
$$

(12) for notational brevity:

$$
\begin{aligned}
M^{2}(0, T)= & M^{2}\left(0, T ; R^{n}\right) \times M^{2}\left(0, T ; R^{m}\right) \\
& \times M^{2}\left(0, T ; R^{m \times d}\right) \times l^{2}\left(0, T ; R^{m}\right) .
\end{aligned}
$$

Let us recall more general Ito's formula about càdlàg semimartingales which is important for us to get the maximum principle. Let $X=\left\{X_{t}: t \in[0, T]\right\}$ be càdlàg semimartingales, and we denoted the quadratic variation by $[X]=\left\{[X]_{t}: t \in[0, T]\right\} ; F$ is a $\mathscr{C}^{2}$ real valued function; then $F(X)$ is also semimartingales and following Ito's formula holds:

$$
\begin{aligned}
F\left(X_{t}\right)= & F\left(X_{0}\right)+\int_{0}^{t} F^{\prime}\left(X_{s-}\right) d X_{s} \\
& +\frac{1}{2} \int_{0}^{t} F^{\prime \prime}\left(X_{s}\right) d[X]_{s}^{\mathscr{C}} \\
& +\sum_{0<s \leq t}\left\{F\left(X_{s}\right)-F\left(X_{s-}\right)-F^{\prime}\left(X_{s-}\right) \Delta X_{s}\right\},
\end{aligned}
$$

where $[X]^{\mathscr{C}}$ is the continuous part of the quadratic variation $[X]$.

When $F(X)=X^{2}$ and $F(X)=X_{t} Y_{t}$, where $X, Y$ are two càdlàg semimartingales, we get

$$
\begin{aligned}
X_{t}^{2}= & X_{0}^{2}+\int_{0}^{t} 2 X_{s-} d X_{s}+\int_{0}^{t} d[X]_{s} \\
X_{t} Y_{t}= & X_{0} Y_{0}+\int_{0}^{t} X_{s-} d Y_{s}+\int_{0}^{t} Y_{s-} d X_{s} \\
& +\int_{0}^{t} d[X, Y]_{s}
\end{aligned}
$$

Here $[X, Y]$ is the quadratic covariation of $X, Y$. We can refer to Protter [21] for a complete survey in this topic.

Next, we introduce the existence and uniqueness results for fully coupled FBSDEL (1):

$$
\begin{aligned}
d x_{t}= & b\left(t, x_{t}, y_{t}, z_{t}, r_{t}\right) d t+\sum_{i=1}^{d} \sigma^{i}\left(t, x_{t}, y_{t}, z_{t}, r_{t}\right) d B_{t}^{i} \\
& +\sum_{i=1}^{\infty} g^{i}\left(t, x_{t-}, y_{t-}, z_{t}, r_{t}\right) d H_{t}^{i}, \\
-d y_{t}= & f\left(t, x_{t}, y_{t}, z_{t}, r_{t}\right) d t-\sum_{i=1}^{d} z_{t}^{i} d B_{t}^{i}-\sum_{i=1}^{\infty} r_{t}^{i} d H_{t}^{i}, \\
x_{0}= & a, \\
y_{T}= & \Phi\left(x_{T}\right),
\end{aligned}
$$

where

$$
\begin{aligned}
b & : \Omega \times[0, T] \times R^{n} \times R^{m} \times R^{m \times d} \times l^{2}\left(R^{m}\right) \longrightarrow R^{n}, \\
\sigma: & \Omega \times[0, T] \times R^{n} \times R^{m} \times R^{m \times d} \times l^{2}\left(R^{m}\right) \longrightarrow R^{n \times d}, \\
g & : \Omega \times[0, T] \times R^{n} \times R^{m} \times R^{m \times d} \times l^{2}\left(R^{m}\right) \\
& \longrightarrow l^{2}\left(R^{n}\right), \\
f & : \Omega \times[0, T] \times R^{n} \times R^{m} \times R^{m \times d} \times l^{2}\left(R^{m}\right) \longrightarrow R^{m} .
\end{aligned}
$$


For a given $m \times n$ full rank matrix $G$, we set

$$
\begin{aligned}
\lambda & =\left(\begin{array}{c}
x \\
y \\
z
\end{array}\right), \\
A(t, \lambda, r) & =\left(\begin{array}{c}
-G^{T} f(t, \lambda, r) \\
G b(t, \lambda, r) \\
G \sigma(t, \lambda, r)
\end{array}\right) .
\end{aligned}
$$

Assumption 1. Assume the following.

(i) $b, \sigma, g$, and $f$ are uniformly Lipschitz continuous with respect to $(x, y, z, r)$.

(ii) For each $(\omega, t) \in \Omega \times[0, T], l(\omega, t, 0,0,0,0) \in M^{2}(0, T)$ and $g(\omega, t, 0,0,0,0) \in H^{2}\left(l^{2}\right)$, where $l=b, \sigma, f$, respectively.

(iii) $\Phi(\cdot)$ is uniformly Lipschitz continuous with respect to $x$ and $\forall x, \Phi(x) \in L^{2}\left(\Omega, F_{T}, P\right)$.

Assumption 2. We also assume that

$$
\begin{aligned}
& \left\langle A\left(t, \lambda_{1}, r_{1}\right)-A\left(t, \lambda_{2}, r_{2}\right), \lambda_{1}-\lambda_{2}\right\rangle+\sum_{i=1}^{\infty}\left\langle G \widehat{g^{i}}, \widehat{r^{i}}\right\rangle \\
& \leq-\beta_{1}|G \hat{x}|^{2} \\
& \quad-\beta_{2}\left(\left|G^{T} \hat{y}\right|^{2}+\left|G^{T} \widehat{z}\right|^{2}+\sum_{i=1}^{\infty}\left\|G^{T} \widehat{r^{i}}\right\|^{2}\right) ; \\
& \left\langle\Phi\left(x_{1}\right)-\Phi\left(x_{2}\right), G\left(x_{1}-x_{2}\right)\right\rangle \geq \mu_{1}|G \widehat{x}|^{2},
\end{aligned}
$$

where $\lambda_{1}=\left(x_{1}, y_{1}, z_{1}\right), \lambda_{2}=\left(x_{2}, y_{2}, z_{2}\right), \hat{x}=x_{1}-x_{2}, \hat{y}=$ $y_{1}-y_{2}, \widehat{z}=z_{1}-z_{2}, \widehat{g^{i}}=g^{i}\left(t, \lambda_{1}, r_{1}\right)-g^{i}\left(t, \lambda_{2}, r_{2}\right), \widehat{r^{i}}=r_{1}^{i}-r_{2}^{i}$, and $\beta_{1}, \beta_{2}$ and $\mu_{1}$ are nonnegative constants with $\beta_{1}+\beta_{2}>0$, $\beta_{2}+\mu_{1}>0$. Moreover, we have $\beta_{1}>0, \mu_{1}>0$ (resp., $\beta_{2}>0$ ) when $m>n$ (resp., $n>m$ ). Under Assumptions 1 and 2, in [10], they have got the following lemma.

Lemma 3 (existence and uniqueness theorem of FBSDEL [10]). Under Assumptions 1 and 2, FBSDEL (15) has a unique solution.

\section{Continuity Result Depending on Parameters about FBSDEL}

Next, we are going to get the continuity result depending on parameters about FBSDEL.
Let $\left(b_{\alpha}, \sigma_{\alpha}, g_{\alpha}, f_{\alpha}, \Phi_{\alpha}\right), \alpha \in R$ be a family of FBSDEL:

$$
\begin{aligned}
d x_{t}^{\alpha}= & b_{\alpha}\left(t, x_{t}^{\alpha}, y_{t}^{\alpha}, z_{t}^{\alpha}, r_{t}^{\alpha}\right) d t \\
& +\sum_{i=1}^{d} \sigma_{\alpha}^{i}\left(t, x_{t}^{\alpha}, y_{t}^{\alpha}, z_{t}^{\alpha}, r_{t}^{\alpha}\right) d B_{t}^{i} \\
& +\sum_{i=1}^{\infty} g_{\alpha}^{i}\left(t, x_{t-}^{\alpha}, y_{t-}^{\alpha}, z_{t}^{\alpha}, r_{t}^{\alpha}\right) d H_{t}^{i}, \\
-d y_{t}= & f_{\alpha}\left(t, x_{t}^{\alpha}, y_{t}^{\alpha}, z_{t}^{\alpha}, r_{t}^{\alpha}\right) d t-\sum_{i=1}^{d} z_{t}^{i, \alpha} d B_{t}^{i}
\end{aligned}
$$

$$
-\sum_{i=1}^{\infty} r_{t}^{i, \alpha} d H_{t}^{i}
$$

$$
\begin{aligned}
& x_{0}^{\alpha}=a, \\
& y_{T}^{\alpha}=\Phi_{\alpha}\left(x_{T}^{\alpha}\right) .
\end{aligned}
$$

Assumption 4. (i) The family $\left(b_{\alpha}, \sigma_{\alpha}, g_{\alpha}, f_{\alpha}, \Phi_{\alpha}\right), \alpha \in R$ are equi-Lipschitz with respect to $(x, y, z, r)$ and $x$ separately.

(ii) The function $\alpha \rightarrow\left(b_{\alpha}, \sigma_{\alpha}, g_{\alpha}, f_{\alpha}, \Phi_{\alpha}\right)$ is continuous in their existing space norm sense, respectively.

Then we can get the following continuity result depending on parameters of forward-backward stochastic differential equation driven by Lévy processes.

Theorem 5. Let $\left(b_{\alpha}, \sigma_{\alpha}, g_{\alpha}, f_{\alpha}, \Phi_{\alpha}\right), \alpha \in R$ be a family of FBSDEL satisfying Assumptions 1, 2, and 4 with solutions denoted by $\left(x^{\alpha}, y^{\alpha}, z^{\alpha}, r^{\alpha}\right)$. Thus, the function

$$
\begin{aligned}
& \alpha \longrightarrow\left(x^{\alpha}, y^{\alpha}, z^{\alpha}, r^{\alpha}, x_{T}^{\alpha}\right): \\
& R \longrightarrow M^{2}(0, T) \times L^{2}\left(\Omega, \mathscr{F}_{t}, P, R^{n}\right)
\end{aligned}
$$

is continuous.

Proof. For notational brevity, we only prove the continuity of FBSDEL (19) at $\alpha=0$. Set $\widehat{\lambda}_{t}=\lambda_{t}^{\alpha}-\lambda_{t}^{0}=\left(x_{t}^{\alpha}-x_{t}^{0}, y_{t}^{\alpha}-y_{t}^{0}, z_{t}^{\alpha}-\right.$ $\left.z_{t}^{0}\right)$ and $\widehat{r}_{t}=r_{t}^{\alpha}-r_{t}^{0}$. From Assumptions 1, 2, and 4, applying the usual technique to $\widehat{x}_{t}$ of Itô's SDE with Lévy process, we can get

$$
\begin{aligned}
\sup _{0 \leq t \leq T} E\left|\widehat{x}_{t}\right|^{2} \leq & K_{1} E \int_{0}^{T}\left(\left|\widehat{y}_{t}\right|^{2}+\left|\widehat{z}_{t}\right|^{2}+\left\|\widehat{r}_{t}^{i}\right\|^{2}\right) d t \\
& +K_{1} E \int_{0}^{T}\left(\left|\widehat{b}_{t}\right|^{2}+\left|\widehat{\sigma}_{t}\right|^{2}+\left\|\widehat{g}_{t}\right\|^{2}\right) d t .
\end{aligned}
$$

Applying the same technique to $\widehat{y}_{t}$ of BSDE with Lévy process, then

$$
\begin{aligned}
& E \int_{0}^{T}\left(\left|\widehat{y}_{t}\right|^{2}+\left|\widehat{z}_{t}\right|^{2}+\left\|\widehat{r}_{t}^{i}\right\|^{2}\right) d t \\
& \quad \leq K_{1}\left(E \int_{0}^{T}\left|\widehat{x}_{t}\right|^{2} d t+E\left|\widehat{x}_{T}\right|^{2}+E \int_{0}^{T}\left|\widehat{f}_{t}\right|^{2} d t\right. \\
& \left.\quad+E\left|\Phi_{\alpha}\left(x_{T}^{0}\right)-\Phi_{0}\left(x_{T}^{0}\right)\right|^{2}\right) .
\end{aligned}
$$


Here $K_{1}, K_{2}$ depend on the Lipschitz constants and $T$, and

$$
\begin{aligned}
& \widehat{f}_{t}=f_{\alpha}\left(t, \lambda_{t}^{0}, r_{t}^{0}\right)-f_{0}\left(t, \lambda_{t}^{0}, r_{t}^{0}\right), \\
& \widehat{b}_{t}=b_{\alpha}\left(t, \lambda_{t}^{0}, r_{t}^{0}\right)-b_{0}\left(t, \lambda_{t}^{0}, r_{t}^{0}\right), \\
& \widehat{\sigma}_{t}=\sigma_{\alpha}\left(t, \lambda_{t}^{0}, r_{t}^{0}\right)-\sigma_{0}\left(t, \lambda_{t}^{0}, r_{t}^{0}\right), \\
& \widehat{g}_{t}=g_{\alpha}\left(t, \lambda_{t}^{0}, r_{t}^{0}\right)-g_{0}\left(t, \lambda_{t}^{0}, r_{t}^{0}\right) .
\end{aligned}
$$

Set

$$
A_{\alpha}(t, \lambda, r)=\left(\begin{array}{c}
-G^{T} f_{\alpha}(t, \lambda, r) \\
G b_{\alpha}(t, \lambda, r) \\
G \sigma_{\alpha}(t, \lambda, r)
\end{array}\right),
$$

and applying Itô's formula to $\left\langle G \widehat{x}_{t}, \widehat{y}_{t}\right\rangle$ yields

$$
\begin{aligned}
E & \left\langle\Phi_{\alpha}\left(x_{T}^{\alpha}\right)-\Phi_{\alpha}\left(x_{T}^{0}\right), G \widehat{x}_{T}\right\rangle+E\left\langle\Phi_{0}\left(x_{T}^{\alpha}\right)\right. \\
& \left.-\Phi_{0}\left(x_{T}^{0}\right), G \widehat{x}_{T}\right\rangle \\
& =E \int_{0}^{T}\left[\left\langle A_{\alpha}\left(t, \lambda_{t}^{\alpha}, r_{t}^{\alpha}\right)-A_{\alpha}\left(t, \lambda_{t}^{0}, r_{t}^{0}\right), \hat{\lambda}_{t}\right\rangle\right. \\
& \left.+\sum_{i=1}^{\infty}\left\langle G\left(g_{\alpha}^{i}\left(t, \lambda_{t}^{\alpha}, r_{t}^{\alpha}\right)-g_{\alpha}^{i}\left(t, \lambda_{t}^{0}, r_{t}^{0}\right)\right), \widehat{r}_{t}^{i}\right\rangle\right] d t \\
& +E \int_{0}^{T}\left[\left\langle\hat{x}_{t},-G^{T} \widehat{f}_{t}\right\rangle+\left\langle G^{T} \widehat{y}_{t}, \widehat{b}_{t}\right\rangle+\sum_{i=1}^{d}\left\langle\widehat{z}_{t}^{i}, G \widehat{\sigma}_{t}^{i}\right\rangle\right. \\
& \left.+\sum_{i=1}^{\infty}\left\langle G^{T} \hat{r}_{t}^{i}, \hat{g}_{t}^{i}\right\rangle\right] d t .
\end{aligned}
$$

From the above three estimates, we get

$$
\begin{aligned}
& \beta_{1} E \int_{0}^{T}\left|G \widehat{x}_{t}\right|^{2} d t+\mu_{1} E\left|G \widehat{x}_{T}\right|^{2} \\
& +\beta_{2} E \int_{0}^{T}\left[\left|G^{T} \widehat{y}_{t}\right|^{2}+\left|G^{T} \widehat{z}_{t}\right|^{2}+\left\|G^{T} \widehat{r}_{t}\right\|^{2}\right] d t \\
& \leq C_{1}\left[E\left|\Phi_{\alpha}\left(x_{T}^{0}\right)-\Phi_{0}\left(x_{T}^{0}\right)\right|^{2}\right. \\
& \left.+E \int_{0}^{T}\left(\left|\widehat{f}_{t}\right|^{2}+\left|\widehat{b}_{t}\right|^{2}+\left|\widehat{\sigma}_{t}\right|^{2}+\left\|\widehat{g}_{t}\right\|^{2}\right) d t\right] \\
& \left.+\delta E\left|\widehat{x}_{T}\right|^{2}\right] \\
& +\delta E \int_{0}^{T}\left(\left|\widehat{x}_{t}\right|^{2}+\left|\widehat{y}_{t}\right|^{2}+\left|\widehat{z}_{t}\right|^{2}+\left\|\widehat{r}_{t}\right\|^{2}\right) d t,
\end{aligned}
$$

where the constant $C_{1}$ depends on the Lipschitz constants $T$ and $\delta$. When $m \geq n, \beta_{1}>0$, and $\mu_{1}>0$, then

$$
\delta=\min \left(\frac{1}{3}, \frac{1}{3 K_{1}}, \frac{\beta_{1}\left|G^{T} G\right|}{3}, \frac{\mu_{1}\left|G^{T} G\right|}{3}\right) .
$$

If $m \leq n, \beta_{2}>0$, and $\mu_{1} \geq 0$, then

$$
\delta=\min \left(\frac{1}{3}, \frac{1}{3 K_{1}}, \frac{1}{3 K_{1} T}, \frac{\beta_{2}\left|G G^{T}\right|}{3}\right) .
$$

Thus, it is clear whatever $\beta_{1}>0, \beta_{2} \geq 0$, and $\mu_{1}>0$ or $\beta_{1} \geq 0, \beta_{2}>0$, and $\mu_{1} \geq 0$ we always have

$$
\begin{aligned}
& E\left|\widehat{x}_{T}\right|^{2}+E \int_{0}^{T}\left(\left|\widehat{\lambda}_{t}\right|^{2}+\left\|\widehat{r}_{t}\right\|^{2}\right) d t \\
& \leq C\left[E\left|\Phi_{\alpha}\left(x_{T}^{0}\right)-\Phi_{0}\left(x_{T}^{0}\right)\right|^{2}\right. \\
& \left.+E \int_{0}^{T}\left(\left|\widehat{f}_{t}\right|^{2}+\left|\widehat{b}_{t}\right|^{2}+\left|\widehat{\sigma}_{t}\right|^{2}+\left\|\widehat{g}_{t}\right\|^{2}\right)\right] .
\end{aligned}
$$

The proof is completed.

\section{Maximum Principle}

Let us consider the following full coupled forward-backward stochastic control system:

$$
\begin{aligned}
d x_{t}= & b\left(t, x_{t}, y_{t}, z_{t}, r_{t}, u_{t}\right) d t \\
& +\sum_{i=1}^{d} \sigma^{i}\left(t, x_{t}, y_{t}, z_{t}, u_{t}\right) d B_{t}^{i} \\
& +\sum_{i=1}^{\infty} g^{i}\left(t, x_{t-}, y_{t-}, r_{t}, u_{t}\right) d H_{t}^{i}, \\
-d y_{t}= & f\left(t, x_{t}, y_{t}, z_{t}, r_{t}, u_{t}\right) d t-\sum_{i=1}^{d} z_{t}^{i} d B_{t}^{i}-\sum_{i=1}^{\infty} r_{t}^{i} d H_{t}^{i}, \\
x_{0}= & a, \\
y_{T}= & \Phi\left(x_{T}\right),
\end{aligned}
$$

where $\left(x_{t}, y_{t}, z_{t}, r_{t}\right)$ take values in $R^{n} \times R^{m} \times R^{m \times d} \times l^{2}\left(R^{m}\right)$; $a \in R^{n}$ is given.

Let $U$ be a nonempty convex subset of $R^{k}$. We define the admissible control set $U_{a d}=\left\{u(\cdot) \in M^{2}\left(0, T ; R^{k}\right) ; u_{t} \in U, 0 \leq\right.$ $t \leq T$, a.e., a.s. $\}$ and the cost functional:

$$
J(u)=E \int_{0}^{T} L\left(x_{t}, y_{t}, z_{t}, r_{t}, u_{t}\right) d t+h\left(x_{T}\right)+\gamma\left(y_{0}\right) .
$$

The optimal control problem is to find $\bar{u} \in U_{a d}$, such that

$$
J(\bar{u}(\cdot))=\inf _{u(\cdot) \in U[0, T]} J(u(\cdot)) .
$$

Assumption 6. Now we introduce the basic assumptions of this section as follows.

(i) $b, f$, and $L$ are continuously differentiable with respect to $(x, y, z, r, u) ; \sigma$ is continuously differentiable with respect to $(x, y, z, u) ; g$ is continuously differentiable with respect to $(x, y, r, u) ; \Phi$ and $h$ are continuously differentiable with respect to $x ; \gamma$ is continuously differentiable with respect to $y$. And the derivatives of each function are all bounded. 
(ii) For each $(x, y, z, r, u) \in R^{n} \times R^{m} \times R^{m \times d} \times l^{2}\left(R^{m}\right) \times U_{a d}$, there exists a constant $C>0$, such that

$$
\begin{aligned}
&|L| \leq C\left(1+|x|^{2}+|y|^{2}+|z|^{2}+\|r\|^{2}+|u|^{2}\right), \\
&|h| \leq C\left(1+|x|^{2}\right), \\
&|\gamma| \leq\left(1+|y|^{2}\right), \\
&\left|L_{x}\right|+\left|L_{y}\right|+\left|L_{z}\right|+\left|L_{r}\right|+\left|L_{u}\right| \\
& \quad \leq C(1+|x|+|y|+|z|+\|r\|+|u|), \\
&\left|h_{x}\right| \leq C(1+|x|), \\
&\left|\gamma_{y}\right| \leq C(1+|y|) .
\end{aligned}
$$

(iii) For any given admissible control $u(\cdot)$, (30) satisfies Assumptions 1 and 2.

Then, for a given admissible control, from Lemma 3, there exists a unique solution satisfying control system (30).

Let $\bar{u}_{t}$ be an optimal control and let $\left(\bar{x}_{t}, \bar{y}_{t}, \bar{z}_{t}, \bar{r}_{t}\right)$ be the corresponding trajectory. For any given admissible control $u_{t}$ and $0 \leq \varepsilon \leq 1$, we define

$$
u_{t}^{\varepsilon}=\bar{u}_{t}+\varepsilon\left(u_{t}-\bar{u}_{t}\right) .
$$

Since $U_{a d}$ is convex, then $u_{t}^{\varepsilon}$ is in $U_{a d}$; that is, $u_{t}^{\varepsilon}$ is an admissible control and $\left(x_{t}^{\varepsilon}, y_{t}^{\varepsilon}, z_{t}^{\varepsilon}, r_{t}^{\varepsilon}\right)$ is the corresponding trajectory.

We introduce the following variational equation:

$$
\begin{aligned}
& d X_{t}=\left[b_{x}(t) X_{t}+b_{y}(t) Y_{t}+b_{z}(t) Z_{t}+b_{r}(t) R_{t}\right. \\
& \left.+b_{u}(t)\left(u_{t}-\bar{u}_{t}\right)\right] d t+\sum_{i=1}^{d}\left[\sigma_{x}^{i}(t) X_{t}+\sigma_{y}^{i}(t) Y_{t}\right. \\
& \left.+\sigma_{z}^{i}(t) Z_{t}+\sigma_{u}^{i}(t)\left(u_{t}-\bar{u}_{t}\right)\right] d B_{t}^{i}+\sum_{i=1}^{\infty}\left[g_{x}^{i}(t) X_{t-}\right. \\
& \left.+g_{y}^{i}(t) Y_{t-}+g_{r}^{i}(t) R_{t}+g_{u}^{i}(t)\left(u_{t}-\bar{u}_{t}\right)\right] d H_{t}^{i}, \\
& -d y_{t}=\left[f_{x}(t) X_{t}+f_{y}(t) Y_{t}+f_{z}(t) Z_{t}+f_{r}(t) R_{t}\right. \\
& \left.\quad+f_{u}^{i}(t)\left(u_{t}-\bar{u}_{t}\right)\right] d t-\sum_{i=1}^{d} Z_{t}^{i} d B_{t}^{i}-\sum_{i=1}^{\infty} R_{t}^{i} d H_{t}^{i}, \\
& X_{0}=0, \\
& Y_{T}=\Phi_{x}\left(\bar{x}_{t}\right) X_{T} .
\end{aligned}
$$

From Assumption 6, we can verify that variational equation (35) satisfies Lemma 3. Thus, there exists a unique solution $\left(X_{t}, Y_{t}, Z_{t}, R_{t}\right)$ satisfying variational equation. In order to get the maximum principle, we also need the following lemma.
Lemma 7. Assume that Assumption 6 holds. We have

$$
\begin{aligned}
& E \sup _{0 \leq t \leq T}\left|x_{t}^{\varepsilon}-\bar{x}_{t}-\varepsilon X_{t}\right|^{2}=o\left(\varepsilon^{2}\right), \\
& E \sup _{0 \leq t \leq T}\left|y_{t}^{\varepsilon}-\bar{y}_{t}-\varepsilon Y_{t}\right|^{2}+E \int_{0}^{T}\left|z_{t}^{\varepsilon}-\bar{z}_{t}-\varepsilon Z_{t}\right|^{2} d t \\
& \quad+E \int_{0}^{T}\left\|r_{t}^{\varepsilon}-\bar{r}_{t}-\varepsilon R_{t}\right\|^{2} d t=o\left(\varepsilon^{2}\right),
\end{aligned}
$$

where $\left(X_{t}, Y_{t}, Z_{t}, R_{t}\right)$ is the solution of variational equation (35).

Proof. Set

$$
\begin{aligned}
& \Delta x_{t}=\varepsilon^{-1}\left(x_{t}^{\varepsilon}-\bar{x}_{t}\right), \\
& \Delta y_{t}=\varepsilon^{-1}\left(y_{t}^{\varepsilon}-\bar{y}_{t}\right), \\
& \Delta z_{t}=\varepsilon^{-1}\left(z_{t}^{\varepsilon}-\bar{z}_{t}\right), \\
& \Delta r_{t}=\varepsilon^{-1}\left(r_{t}^{\varepsilon}-\bar{r}_{t}\right),
\end{aligned}
$$

and then

$$
\begin{aligned}
& d \Delta x_{t}=\varepsilon^{-1}\left[b\left(t, x_{t}^{\varepsilon}, y_{t}^{\varepsilon}, z_{t}^{\varepsilon}, r_{t}^{\varepsilon}, u_{t}^{\varepsilon}\right)\right. \\
& \left.-b\left(t, \bar{x}_{t}, \bar{y}_{t}, \bar{z}_{t}, \bar{r}_{t}, \bar{u}_{t}\right)\right] d t \\
& +\varepsilon^{-1}\left[\sum_{i=1}^{d} \sigma^{i}\left(t, x_{t}^{\varepsilon}, y_{t}^{\varepsilon}, z_{t}^{\varepsilon}, r_{t}^{\varepsilon}, u_{t}^{\varepsilon}\right)\right. \\
& \left.-\sum_{i=1}^{d} \sigma^{i}\left(t, \bar{x}_{t}, \bar{y}_{t}, \bar{z}_{t}, \bar{r}_{t}, \bar{u}_{t}\right)\right] d B_{t}^{i} \\
& +\varepsilon^{-1}\left[\sum_{i=1}^{\infty} g^{i}\left(t, x_{t-}^{\varepsilon}, y_{t-}^{\varepsilon}, z_{t}^{\varepsilon}, r_{t}^{\varepsilon}, u_{t}^{\varepsilon}\right)\right. \\
& \left.-\sum_{i=1}^{\infty} g^{i}\left(t, \bar{x}_{t-}, \bar{y}_{t-}, \bar{z}_{t}, \bar{r}_{t}, \bar{u}_{t}\right)\right] d H_{t}^{i}, \\
& -d \Delta y_{t}=\varepsilon^{-1}\left[f\left(t, x_{t}^{\varepsilon}, y_{t}^{\varepsilon}, z_{t}^{\varepsilon}, r_{t}^{\varepsilon}, u_{t}^{\varepsilon}\right)\right. \\
& \left.-f\left(t, \bar{x}_{t}, \bar{y}_{t}, \bar{z}_{t}, \bar{r}_{t}, \bar{u}_{t}\right)\right] d t-\sum_{i=1}^{d} \Delta z_{t}^{i} d B_{t}^{i} \\
& \quad-\sum_{i=1}^{\infty} \Delta r_{t}^{i} d H_{t}^{i}, \\
& \Delta x_{0}=0, \\
& \Delta y_{T}=\varepsilon^{-1}\left[\Phi\left(x_{T}^{\varepsilon}\right)-\Phi\left(\bar{x}_{T}\right)\right] .
\end{aligned}
$$


We can transform (38) into

$d \Delta x_{t}$

$$
\begin{aligned}
& =\widehat{b}\left(t, \Delta x_{t}, \Delta y_{t}, \Delta z_{t}, \Delta r_{t}, u_{t}-\bar{u}_{t}\right) d t \\
& \quad+\sum_{i=1}^{d} \widehat{\sigma}^{i}\left(t, \Delta x_{t}, \Delta y_{t}, \Delta z_{t}, \Delta r_{t}, u_{t}-\bar{u}_{t}\right) d B_{t}^{i} \\
& \quad+\sum_{i=1}^{\infty} \widehat{g}^{i}\left(t, \Delta x_{t-}, \Delta y_{t-}, \Delta z_{t}, \Delta r_{t}, u_{t}-\bar{u}_{t}\right) d H_{t}^{i}, \\
& -d \Delta y_{t} \\
& =\widehat{f}\left(t, \Delta x_{t}, \Delta y_{t}, \Delta z_{t}, \Delta r_{t}, u_{t}-\bar{u}_{t}\right) d t-\sum_{i=1}^{d} \Delta z_{t}^{i} d B_{t}^{i} \\
& \quad-\sum_{i=1}^{\infty} \Delta r_{t}^{i} d H_{t}^{i},
\end{aligned}
$$$$
\Delta x_{0}=0 \text {, }
$$$$
\Delta y_{T}=\varepsilon^{-1}\left[\Phi\left(x_{T}^{\varepsilon}\right)-\Phi\left(\bar{x}_{T}\right)\right],
$$

where

$$
\begin{aligned}
& \hat{l}\left(t, \bar{x}_{t}, \bar{y}_{t}, \bar{z}_{t}, \bar{r}_{t}, u_{t}-\bar{u}_{t}\right) \\
& =A^{l}(t) \bar{x}_{t}+B^{l}(t) \bar{y}_{t}+C^{l}(t) \bar{z}_{t}+D^{l}(t) \bar{r}_{t} \\
& \quad+E^{l}(t)\left(u_{t}-\bar{u}_{t}\right),
\end{aligned}
$$

for $l=b, \sigma, g, f$, respectively, and

$$
\begin{aligned}
& A^{l}(t)= \begin{cases}A(t), & x_{t}^{\varepsilon}-\bar{x}_{t} \neq 0 \\
0, & \text { otherwise }\end{cases} \\
& B^{l}(t)= \begin{cases}B(t), & y_{t}^{\varepsilon}-\bar{y}_{t} \neq 0 \\
0, & \text { otherwise }\end{cases} \\
& C^{l}(t)= \begin{cases}C(t), & z_{t}^{\varepsilon}-\bar{z}_{t} \neq 0 \\
0, & \text { otherwise }\end{cases} \\
& D^{l}(t)= \begin{cases}D(t), & r_{t}^{\varepsilon}-\bar{r}_{t} \neq 0 \\
0, & \text { otherwise }\end{cases} \\
& E^{l}(t)= \begin{cases}E(t), & u_{t}-\bar{u}_{t} \neq 0 \\
0, & \text { otherwise }\end{cases}
\end{aligned}
$$

where

$$
\begin{aligned}
& A(t)=\left(x_{t}^{\varepsilon}-\bar{x}_{t}\right)^{-1} \\
& \cdot\left[l\left(t, x_{t}^{\varepsilon}, y_{t}^{\varepsilon}, z_{t}^{\varepsilon}, r_{t}^{\varepsilon}, u_{t}^{\varepsilon}\right)-l\left(t, \bar{x}_{t}, y_{t}^{\varepsilon}, z_{t}^{\varepsilon}, r_{t}^{\varepsilon}, u_{t}^{\varepsilon}\right)\right], \\
& B(t)=\left(y_{t}^{\varepsilon}-\bar{y}_{t}\right)^{-1} \\
& \cdot\left[l\left(t, \bar{x}_{t}, y_{t}^{\varepsilon}, z_{t}^{\varepsilon}, r_{t}^{\varepsilon}, u_{t}^{\varepsilon}\right)-l\left(t, \bar{x}_{t}, \bar{y}_{t}, z_{t}^{\varepsilon}, r_{t}^{\varepsilon}, u_{t}^{\varepsilon}\right)\right], \\
& C(t)=\left(z_{t}^{\varepsilon}-\bar{z}_{t}\right)^{-1} \\
& \cdot\left[l\left(t, \bar{x}_{t}, \bar{y}_{t}, z_{t}^{\varepsilon}, r_{t}^{\varepsilon}, u_{t}^{\varepsilon}\right)-l\left(t, \bar{x}_{t}, \bar{y}_{t}, \bar{z}_{t}, r_{t}^{\varepsilon}, u_{t}^{\varepsilon}\right)\right], \\
& D(t)=\left(r_{t}^{\varepsilon}-\bar{r}_{t}\right)^{-1} \\
& \cdot\left[l\left(t, \bar{x}_{t}, \bar{y}_{t}, \bar{z}_{t}, r_{t}^{\varepsilon}, u_{t}^{\varepsilon}\right)-l\left(t, \bar{x}_{t}, \bar{y}_{t}, \bar{z}_{t}, \bar{r}_{t}, u_{t}^{\varepsilon}\right)\right], \\
& E(t)=\left[\varepsilon\left(u_{t}-\bar{u}_{t}\right)\right]^{-1} \\
& \cdot\left[l\left(t, \bar{x}_{t}, \bar{y}_{t}, \bar{z}_{t}, \bar{r}_{t}, u_{t}^{\varepsilon}\right)-l\left(t, \bar{x}_{t}, \bar{y}_{t}, \bar{z}_{t}, \bar{r}_{t}, \bar{u}_{t}\right)\right] .
\end{aligned}
$$

From the continuity result depending on parameters we have got in Section 3, we know that

$$
\begin{aligned}
& E \sup _{0 \leq t \leq T}\left|x_{t}^{\varepsilon}-\bar{x}_{t}\right|^{2}+E \sup _{0 \leq t \leq T}\left|y_{t}^{\varepsilon}-\bar{y}_{t}\right|^{2} \\
& \quad+E \int_{0}^{t}\left|z_{t}^{\varepsilon}-\bar{z}_{t}\right|^{2} d t+E \int_{0}^{t}\left\|r_{t}^{\varepsilon}-\bar{r}_{t}\right\|^{2} d t \leq K \varepsilon^{2} ;
\end{aligned}
$$

that is, $\left(x_{t}^{\varepsilon}-\bar{x}_{t}, y_{t}^{\varepsilon}-\bar{y}_{t}, z_{t}^{\varepsilon}-\bar{z}_{t}, r_{t}^{\varepsilon}-\bar{r}_{t}\right) \rightarrow 0$ in $M^{2}(0, T)$, as $\varepsilon \rightarrow 0$. Together with Assumption 6, we can get

$$
\begin{aligned}
& \lim _{\varepsilon \rightarrow 0} A^{l}(t)=l_{x}\left(t, \bar{x}_{t}, \bar{y}_{t}, \bar{z}_{t}, \bar{r}_{t}, \bar{u}_{t}\right), \\
& \lim _{\varepsilon \rightarrow 0} B^{l}(t)=l_{y}\left(t, \bar{x}_{t}, \bar{y}_{t}, \bar{z}_{t}, \bar{r}_{t}, \bar{u}_{t}\right), \\
& \lim _{\varepsilon \rightarrow 0} C^{l}(t)=l_{z}\left(t, \bar{x}_{t}, \bar{y}_{t}, \bar{z}_{t}, \bar{r}_{t}, \bar{u}_{t}\right), \\
& \lim _{\varepsilon \rightarrow 0} D^{l}(t)=l_{r}\left(t, \bar{x}_{t}, \bar{y}_{t}, \bar{z}_{t}, \bar{r}_{t}, \bar{u}_{t}\right), \\
& \lim _{\varepsilon \rightarrow 0} E^{l}(t)=l_{u}\left(t, \bar{x}_{t}, \bar{y}_{t}, \bar{z}_{t}, \bar{r}_{t}, \bar{u}_{t}\right) \\
& \lim _{\varepsilon \rightarrow 0}\left(t, \Delta x_{t}, \Delta y_{t}, \Delta z_{t}, \Delta r_{t}, u_{t}-\bar{u}_{t}\right) \\
& =l_{x}\left(t, \bar{x}_{t}, \bar{y}_{t}, \bar{z}_{t}, \bar{r}_{t}, \bar{u}_{t}\right) \Delta x_{t} \\
& \quad+l_{y}\left(t, \bar{x}_{t}, \bar{y}_{t}, \bar{z}_{t}, \bar{r}_{t}, \bar{u}_{t}\right) \Delta y_{t} \\
& \quad+l_{z}\left(t, \bar{x}_{t}, \bar{y}_{t}, \bar{z}_{t}, \bar{r}_{t}, \bar{u}_{t}\right) \Delta z_{t} \\
& \quad+l_{r}\left(t, \bar{x}_{t}, \bar{y}_{t}, \bar{z}_{t}, \bar{r}_{t}, \bar{u}_{t}\right) \Delta r_{t} \\
& +l_{u}\left(t, \bar{x}_{t}, \bar{y}_{t}, \bar{z}_{t}, \bar{r}_{t}, \bar{u}_{t}\right)\left(u_{t}-\bar{u}_{t}\right) .
\end{aligned}
$$

As FBSDEL (35) has a unique solution $\left(X_{t}, Y_{t}, Z_{t}, R_{t}\right)$ under Assumption 6, from the continuity and uniqueness result by Lemma 3 , we know that $\left(\Delta x_{t}, \Delta y_{t}, \Delta z_{t}, \Delta r_{t}\right)$ converges to $\left(X_{t}, Y_{t}, Z_{t}, R_{t}\right)$ in $M^{2}([0, T])$ as $\varepsilon \rightarrow 0$.

The proof is complete. 
Notice that $\lim _{\varepsilon \rightarrow 0^{+}}\left(J\left(u_{t}^{\varepsilon}-J\left(\bar{u}_{t}\right)\right) / \varepsilon\right) \geq 0$ and we can get the following variational inequality.

Lemma 8. Assume that Assumption 6 holds; then

$$
\begin{aligned}
& E \int_{0}^{T} L_{x}(t) X_{t} d t+E \int_{0}^{T} L_{y}(t) Y_{t} d t \\
& \quad+E \int_{0}^{T} L_{z}(t) Z_{t} d t+E \int_{0}^{T} L_{r}(t) R_{t} d t \\
& \quad+E \int_{0}^{T} L_{u}(t)\left(u_{t}-\bar{u}_{t}\right) d t+E h_{x}\left(x_{T}\right) X_{T} \\
& \quad+E \gamma_{y}\left(y_{0}\right) Y_{0} \geq 0 .
\end{aligned}
$$

Proof. First we have

$$
\begin{aligned}
& \frac{J\left(u_{t}^{\varepsilon}-J\left(\bar{u}_{t}\right)\right)}{\varepsilon}=\frac{1}{\varepsilon}\left\{E \int _ { 0 } ^ { T } \left[L\left(t, x_{t}^{\varepsilon}, y_{t}^{\varepsilon}, z_{t}^{\varepsilon}, r_{t}^{\varepsilon}, u_{t}^{\varepsilon}\right)\right.\right. \\
& \left.-L\left(t, \bar{x}_{t}, \bar{y}_{t}, \bar{z}_{t}, \bar{r}_{t}, \bar{u}_{t}\right)\right] d t+E\left[h\left(x_{T}^{\varepsilon}\right)-h\left(\bar{x}_{T}\right)\right] \\
& \left.+E\left[\gamma\left(y_{0}^{\varepsilon}\right)-\gamma\left(\bar{y}_{0}\right)\right]\right\} .
\end{aligned}
$$

Under Assumption 6, from Lemma 7, we can get

$$
\begin{aligned}
& \lim _{\varepsilon \rightarrow 0} \frac{1}{\varepsilon} E \int_{0}^{T}\left[L\left(t, x_{t}^{\varepsilon}, y_{t}^{\varepsilon}, z_{t}^{\varepsilon}, r_{t}^{\varepsilon}, u_{t}^{\varepsilon}\right)\right. \\
&\left.-L\left(t, \bar{x}_{t}, \bar{y}_{t}, \bar{z}_{t}, \bar{r}_{t}, \bar{u}_{t}\right)\right] d t \\
& \longrightarrow E \int_{0}^{T}\left[L_{x}\left(t, \bar{x}_{t}, \bar{y}_{t}, \bar{z}_{t}, \bar{r}_{t}, \bar{u}_{t}\right) X_{t}\right. \\
&+L_{y}\left(t, \bar{x}_{t}, \bar{y}_{t}, \bar{z}_{t}, \bar{r}_{t}, \bar{u}_{t}\right) Y_{t} \\
&+L_{z}\left(t, \bar{x}_{t}, \bar{y}_{t}, \bar{z}_{t}, \bar{r}_{t}, \bar{u}_{t}\right) Z_{t} \\
&+L_{r}\left(t, \bar{x}_{t}, \bar{y}_{t}, \bar{z}_{t}, \bar{r}_{t}, \bar{u}_{t}\right) R_{t} \\
&\left.+L_{u}\left(t, \bar{x}_{t}, \bar{y}_{t}, \bar{z}_{t}, \bar{r}_{t}, \bar{u}_{t}\right)\left(u_{t}-\bar{u}_{t}\right)\right] d t, \\
& \lim _{\varepsilon \rightarrow 0} \frac{1}{\varepsilon} E\left[h\left(x_{T}^{\varepsilon}\right)-h\left(\bar{x}_{T}\right)\right] \longrightarrow E\left[h_{x}\left(\bar{x}_{T}\right) X_{T}\right], \\
& \lim _{\varepsilon \rightarrow 0} \frac{1}{\varepsilon} E\left[\gamma\left(y_{0}^{\varepsilon}\right)-\gamma\left(\bar{y}_{0}\right)\right] \longrightarrow E\left[\gamma_{y}\left(\bar{y}_{0}\right) Y_{0}\right] .
\end{aligned}
$$

Then (45) is proved.

The proof is complete.

We define the Hamiltonian function $H$ as follows:

$$
\begin{aligned}
& H(t, x, y, z, r, u, p, q, k, \rho) \\
&=\langle p,-f(t, x, y, z, r, u)\rangle+\langle q, b(t, x, y, z, r, u)\rangle \\
&+\langle k, \sigma(t, x, y, z, u)\rangle+\langle\rho, g(t, x, y, r, u)\rangle \\
&+L(t, x, y, z, r, u),
\end{aligned}
$$

and the following adjoint forward-backward equation to variational equation (35):

$$
\begin{aligned}
d p_{t} & \\
= & -H_{y}\left(t, x_{t}, y_{t}, z_{t}, r_{t}, u_{t}, p_{t}, q_{t}, k_{t}, \rho_{t}\right) d t \\
& -\sum_{i=1}^{d} H_{z}^{i}\left(t, x_{t}, y_{t}, z_{t}, r_{t}, u_{t}, p_{t}, q_{t}, k_{t}, \rho_{t}\right) d B_{t}^{i} \\
& \quad-\sum_{i=1}^{\infty} H_{r}^{i}\left(t, x_{t-}, y_{t-}, z_{t}, r_{t}, u_{t}, p_{t}, q_{t}, k_{t}, \rho_{t}\right) d H_{t}^{i}, \\
-d q_{t} & \\
= & H_{x}\left(t, x_{t}, y_{t}, z_{t}, r_{t}, u_{t}, p_{t}, q_{t}, k_{t}, \rho_{t}\right) d t-\sum_{i=1}^{d} k_{t}^{i} d B_{t}^{i} \\
& -\sum_{i=1}^{\infty} \rho_{t}^{i} d H_{t}^{i}, \\
p_{0}= & -\gamma_{y}\left(y_{0}\right), \\
q_{T}= & h_{x}\left(x_{T}\right)-\Phi_{x}\left(x_{T}\right) p_{T} .
\end{aligned}
$$

It is easy to verify that (49) satisfies Assumptions 1 and 2 ; then there exists a unique quarter $\left(p_{t}, q_{t}, k_{t}, \rho_{t}\right)$ satisfying (49).

Then we have the main result of this paper which is the following theorem.

Theorem 9. Supposing that Assumptions 1, 2, 4, and 6 hold, $\left(\bar{x}_{t}, \bar{y}_{t}, \bar{z}_{t}, \bar{r}_{t}, \bar{u}_{t}\right)$ is an optimal pair for our optimal control problem and $\left(p_{t}, q_{t}, k_{t}, \rho_{\mathrm{t}}\right)$ is the solution to corresponding adjoint equation (49). Then for each admissible control $u_{t} \in$ $U(0, T)$ we have

$$
\begin{array}{r}
\left\langle H_{u}\left(t, \bar{x}_{t}, \bar{y}_{t}, \bar{z}_{t}, \bar{r}_{t}, \bar{u}_{t}, p_{t}, q_{t}, k_{t}, \rho_{t}\right),\left(u_{t}-\bar{u}_{t}\right)\right\rangle \geq 0 \\
\text { a.e., a.s., }
\end{array}
$$

where $H$ is defined by (48).

Proof. Applying Ito's formula to $\left\langle X_{t}, q_{t}\right\rangle+\left\langle Y_{t}, p_{t}\right\rangle$, we can obtain

$$
\begin{aligned}
E & {\left[h_{x}\left(\bar{x}_{T}\right) X_{T}\right]+E\left[\gamma_{y}\left(\bar{y}_{0}\right) Y_{0}\right]+E \int_{0}^{T}\left[L_{x}(t) X_{t}\right.} \\
& +L_{y}(t) Y_{t}+L_{z}(t) Z_{t}+L_{r}(t) R_{t}+L_{u}(t) \\
& \left.\cdot\left(u_{t}-\bar{u}_{t}\right)\right] d t \\
& =E \int_{0}^{T}\left\langle H_{u}\left(t, \bar{x}_{t}, \bar{y}_{t}, \bar{z}_{t}, \bar{r}_{t}, \bar{u}_{t}, p_{t}, q_{t}, k_{t}, \rho_{t}\right),\right. \\
& \left.\left(u_{t}-\bar{u}_{t}\right)\right\rangle d t .
\end{aligned}
$$


The variational inequality implies for each $u_{t} \in U[0, T]$ that

$$
\begin{aligned}
& E \int_{0}^{T}\left\langle H_{u}\left(t, \bar{x}_{t}, \bar{y}_{t}, \bar{z}_{t}, \bar{r}_{t}, \bar{u}_{t}, p_{t}, q_{t}, k_{t}, \rho_{t}\right),\left(u_{t}-\bar{u}_{t}\right)\right\rangle d t \\
& \quad \geq 0 .
\end{aligned}
$$

The proof is completed.

Next, under some additional convexity conditions, we prove that the maximum principle can be a sufficient condition for optimal control.

Theorem 10. For stochastic control system (30) and the cost functional $J(u)$, if Assumptions 1, 2, 4, and 6 hold, and $y_{T}=M x_{T}, M \in L^{2}\left(\Omega, \mathscr{F}_{T}, P ; R^{m \times n}\right), h$ is convex in $x$, and $\gamma$ is convex in $y$. Let $u_{t}$ be an admissible control and let $\left(x_{t}, y_{t}, z_{t}, r_{t}\right)$ be the corresponding trajectory. Let $\left(p_{t}, q_{t}, k_{t}, \rho_{t}\right)$ be the solution of corresponding adjoint equation (49). Suppose that the Hamiltonian function $H$ is convex in $(x, y, z, r, u)$ and inequality (50) holds; then $u_{t}$ is an optimal control.

Proof. Let $v_{t}$ be an arbitrary admissible control and the corresponding trajectory is $\left(x_{t}^{v}, y_{t}^{v}, z_{t}^{v}, r_{t}^{v}\right)$; then

$$
\begin{aligned}
& J(v(\cdot))-J(u(\cdot)) \\
& =E \int_{0}^{T}\left[L\left(x_{t}^{v}, y_{t}^{v}, z_{t}^{v}, r_{t}^{v}, v_{t}\right)-L\left(x_{t}, y_{t}, z_{t}, r_{t}, u_{t}\right)\right] d t \\
& \quad+E\left[h\left(x_{T}^{v}\right)-h\left(x_{T}\right)\right]+E\left[\gamma\left(y_{0}^{v}\right)-\gamma\left(y_{0}\right)\right] \\
& =I_{1}+I_{2},
\end{aligned}
$$

where

$$
\begin{aligned}
& I_{1} \\
& \quad=E \int_{0}^{T}\left[L\left(x_{t}^{v}, y_{t}^{v}, z_{t}^{v}, r_{t}^{v}, v_{t}\right)-L\left(x_{t}, y_{t}, z_{t}, r_{t}, u_{t}\right)\right] d t, \\
& I_{2}=E\left[h\left(x_{T}^{v}\right)-h\left(x_{T}\right)\right]+E\left[\gamma\left(y_{0}^{v}\right)-\gamma\left(y_{0}\right)\right] .
\end{aligned}
$$

From the definition of Hamiltonian function $H$, we get

$$
\begin{aligned}
I_{1} & =E \int_{0}^{T}\left[H\left(x_{t}^{v}, y_{t}^{v}, z_{t}^{v}, r_{t}^{v}, v_{t}, p_{t}^{v}, q_{t}^{v}, k_{t}^{v}, \rho_{t}^{v}\right)\right. \\
& \left.-H\left(x_{t}, y_{t}, z_{t}, r_{t}, u_{t}, p_{t}, q_{t}, k_{t}, \rho_{t}\right)\right] d t \\
& +E \int_{0}^{T}\left\langle f\left(x_{t}^{v}, y_{t}^{v}, z_{t}^{v}, r_{t}^{v}, v_{t}\right)\right. \\
& \left.-f\left(x_{t}, y_{t}, z_{t}, r_{t}, u_{t}\right), p_{t}\right\rangle d t \\
& -E \int_{0}^{T}\left\langle b\left(x_{t}^{v}, y_{t}^{v}, z_{t}^{v}, r_{t}^{v}, v_{t}\right)\right.
\end{aligned}
$$

$$
\begin{aligned}
& \left.-b\left(x_{t}, y_{t}, z_{t}, r_{t}, u_{t}\right), q_{t}\right\rangle d t-\sum_{i=1}^{d} E \\
& \cdot \int_{0}^{T}\left\langle\sigma^{i}\left(x_{t}^{v}, y_{t}^{v}, z_{t}^{v}, v_{t}\right)-\sigma^{i}\left(x_{t}, y_{t}, z_{t}, u_{t}\right), k_{t}^{i}\right\rangle d t \\
& -\sum_{i=1}^{\infty} E \int_{0}^{T}\left\langle g^{i}\left(x_{t}^{v}, y_{t}^{v}, r_{t}^{v}, v_{t}\right)\right. \\
& \left.-g^{i}\left(x_{t}, y_{t}, \mathrm{r}_{t}, u_{t}\right), \rho_{t}^{i}\right\rangle d t .
\end{aligned}
$$

By convexity of $h, \gamma$ and using Itôs formula to $\left\langle q_{t}, x_{t}^{v}-x_{t}\right\rangle+$ $\left\langle p_{t}, y_{t}^{v}-y_{t}\right\rangle$ we can get

$$
\begin{aligned}
I_{2} & \geq E\left\langle h_{x}\left(x_{T}\right), x_{T}^{v}-x_{T}\right\rangle+E\left\langle\gamma_{y}\left(y_{0}\right), y_{0}^{v}-y_{0}\right\rangle \\
& =-E \int_{0}^{T}\left\langle H_{x}\left(x_{t}, y_{t}, z_{t}, r_{t}, u_{t}, p_{t}, q_{t}, k_{t}, \rho_{\mathrm{t}}\right), x_{t}^{v}\right. \\
& \left.-x_{t}\right\rangle d t \\
& -E \int_{0}^{T}\left\langle H_{y}\left(x_{t}, y_{t}, z_{t}, r_{t}, u_{t}, p_{t}, q_{t}, k_{t}, \rho_{t}\right), y_{t}^{v}\right. \\
& \left.-y_{t}\right\rangle d t-\sum_{i=1}^{d} E \\
& \cdot \int_{0}^{T}\left\langle H_{z}^{i}\left(x_{t}, y_{t}, z_{t}, r_{t}, u_{t}, p_{t}, q_{t}, k_{t}, \rho_{t}\right), z_{t}^{i v}-z_{t}^{i}\right\rangle d t
\end{aligned}
$$

$-\sum_{i=1}^{\infty} E \int_{0}^{T}\left\langle H_{r}^{i}\left(x_{t}, y_{t}, z_{t}, r_{\mathrm{t}}, u_{t}, p_{t}, q_{t}, k_{t}, \rho_{t}\right), r_{t}^{i v}\right.$

$\left.-r_{t}^{i}\right\rangle d t-E \int_{0}^{T}\left\langle f\left(x_{t}^{v}, y_{t}^{v}, z_{t}^{v}, r_{t}^{v}, v_{t}\right)\right.$

$\left.-f\left(x_{t}, y_{t}, z_{t}, r_{t}, u_{t}\right), p_{t}\right\rangle d t$

$+E \int_{0}^{T}\left\langle b\left(x_{t}^{v}, y_{t}^{v}, z_{t}^{v}, r_{t}^{v}, v_{t}\right)\right.$

$\left.-b\left(x_{t}, y_{t}, z_{t}, r_{t}, u_{t}\right), q_{t}\right\rangle d t+\sum_{i=1}^{d} E$

$\cdot \int_{0}^{T}\left\langle\sigma^{i}\left(x_{t}^{v}, y_{t}^{v}, z_{t}^{v}, v_{t}\right)-\sigma^{i}\left(x_{t}, y_{t}, z_{t}, u_{t}\right), k_{t}^{i}\right\rangle d t$

$+\sum_{i=1}^{\infty} E \int_{0}^{T}\left\langle g^{i}\left(x_{t}^{v}, y_{t}^{v}, r_{t}^{v}, v_{t}\right)\right.$

$\left.-g^{i}\left(x_{t}, y_{t}, r_{t}, u_{t}\right), \rho_{t}^{i}\right\rangle d t$, 
and from (53) to (56) we have

$$
\begin{aligned}
& J(v(\cdot))-J(u(\cdot)) \\
& \quad=E \int_{0}^{T}\left[H\left(x_{t}^{v}, y_{t}^{v}, z_{t}^{v}, r_{t}^{v}, v_{t}, p_{t}^{v}, q_{t}^{v}, k_{t}^{v}, \rho_{t}^{v}\right)\right. \\
& \left.\quad-H\left(x_{t}, y_{t}, z_{t}, r_{t}, u_{t}, p_{t}, q_{t}, k_{t}, \rho_{t}\right)\right] d t \\
& \quad-E \int_{0}^{T}\left\langle H_{x}\left(x_{t}, y_{t}, z_{t}, r_{t}, u_{t}, p_{t}, q_{t}, k_{t}, \rho_{t}\right), x_{t}^{v}-x_{t}\right\rangle d t \\
& \quad-E \int_{0}^{T}\left\langle H_{y}\left(x_{t}, y_{t}, z_{t}, r_{t}, u_{t}, p_{t}, q_{t}, k_{t}, \rho_{t}\right), y_{t}^{v}\right. \\
& \left.\quad-y_{t}\right\rangle d t-\sum_{i=1}^{d} E \\
& \quad \int_{0}^{T}\left\langle H_{z}^{i}\left(x_{t}, y_{t}, z_{t}, r_{t}, u_{t}, p_{t}, q_{t}, k_{t}, \rho_{t}\right), z_{t}^{i v}-z_{t}^{i}\right\rangle d t \\
& \quad-\sum_{i=1}^{\infty} E \int_{0}^{T}\left\langle H_{r}^{i}\left(x_{t}, y_{t}, z_{t}, r_{t}, u_{t}, p_{t}, q_{t}, k_{t}, \rho_{t}\right), r_{t}^{i v}\right. \\
& \left.-r_{t}^{i}\right\rangle d t .
\end{aligned}
$$

Moreover, as the Hamiltonian function $H$ is convex in $(x, y, z, r, u)$, the following inequality holds:

$$
\begin{aligned}
& H\left(x_{t}^{v}, y_{t}^{v}, z_{t}^{v}, r_{t}^{v}, v_{t}, p_{t}^{v}, q_{t}^{v}, k_{t}^{v}, \rho_{t}^{v}\right) \\
&- H\left(x_{t}, y_{t}, z_{t}, r_{t}, u_{t}, p_{t}, q_{t}, k_{t}, \rho_{t}\right) \\
& \geq\left\langle H_{x}\left(x_{t}, y_{t}, z_{t}, r_{t}, u_{t}, p_{t}, q_{t}, k_{t}, \rho_{t}\right), x_{t}^{v}-x_{t}\right\rangle \\
&+\left\langle H_{y}\left(x_{t}, y_{t}, z_{t}, r_{t}, u_{t}, p_{t}, q_{t}, k_{t}, \rho_{t}\right), y_{t}^{v}-y_{t}\right\rangle \\
&+\sum_{i=1}^{d}\left\langle H_{z}^{i}\left(x_{t}, y_{t}, z_{t}, r_{t}, u_{t}, p_{t}, q_{t}, k_{t}, \rho_{t}\right), z_{t}^{i v}-z_{t}^{i}\right\rangle \\
&+\sum_{i=1}^{\infty}\left\langle H_{r}^{i}\left(x_{t}, y_{t}, z_{t}, r_{t}, u_{t}, p_{t}, q_{t}, k_{t}, \rho_{t}\right), r_{t}^{i v}-r_{t}^{i}\right\rangle \\
&+\left\langle H_{u}\left(x_{t}, y_{t}, z_{t}, r_{t}, u_{t}, p_{t}, q_{t}, k_{t}, \rho_{t}\right), v_{t}-u_{t}\right\rangle,
\end{aligned}
$$

and from (57) to (58) and together with (50) for arbitrary admissible control $v_{t}$ we have

$$
J(v(\cdot))-J(u(\cdot)) \geq 0,
$$

then admissible control $u_{t}$ is an optimal control.

The proof is completed.

\section{Applications in Linear Quadratic Problem}

In this section, we will apply our stochastic maximum principle to the linear quadratic problem which the linear control system described by fully coupled forward-backward stochastic differential equation driven by Lévy processes:

$$
\begin{aligned}
d x_{t}= & \left(A_{t} x_{t}+B_{t} y_{t}+\sum_{i=1}^{d} C_{t}^{i} z_{t}^{i}+\sum_{i=1}^{\infty} D_{t}^{i} r_{t}^{i}+E_{t} u_{t}\right) d t \\
& +\sum_{i=1}^{d}\left(F_{t}^{i} x_{t}+G_{t}^{i} y_{t}+V_{t}^{i} z_{t}^{i}+W_{t}^{i} u_{t}\right) d B_{t}^{i} \\
& +\sum_{i=1}^{\infty}\left(J_{t}^{i} x_{t_{-}}+K_{t}^{i} y_{t_{-}}+L_{t}^{i} r_{t}^{i}+M_{t}^{i} u_{t}\right) d H_{t}^{i}, \\
-d y_{t}= & \left(N_{t} x_{t}+P_{t} y_{t}+\sum_{i=1}^{d} Q_{t}^{i} z_{t}^{i}+\sum_{i=1}^{\infty} R_{t}^{i} r_{t}^{i}+S_{t} u_{t}\right) d t \\
& -\sum_{i=1}^{d} z_{t}^{i} d B_{t}-\sum_{i=1}^{\infty} r_{t}^{i} d H_{t}^{i}, \\
x_{0}= & a, \\
y_{T}= & h x_{T}+\xi
\end{aligned}
$$

and the cost functional:

$$
\begin{aligned}
& J(u) \\
& =E\left\langle\widetilde{Q} x_{T}, x_{T}\right\rangle+E\left\langle\widetilde{P} y_{0}, y_{0}\right\rangle \\
& \quad+E \int_{0}^{T}\left(\left\langle\widetilde{R}_{t} x_{t}, x_{t}\right\rangle+\left\langle\widetilde{N}_{t} u_{t}, u_{t}\right\rangle+\left\langle\widetilde{L}_{t} y_{t}, y_{t}\right\rangle\right) d t \\
& \quad+\sum_{i=1}^{d} E \int_{0}^{T}\left\langle\widetilde{M}_{t}^{i} z_{t}^{i}, z_{t}^{i}\right\rangle d t+\sum_{i=1}^{\infty} E \int_{0}^{T}\left\langle\widetilde{K}_{t}^{i} r_{t}^{i}, r_{t}^{i}\right\rangle d t,
\end{aligned}
$$

where the $\mathscr{F}_{t}$-predictable matrix processes

$$
\begin{gathered}
A, F^{i}, J^{j}, \widetilde{R}:[0, T] \times \Omega \longrightarrow R^{n \times n}, \\
i=1,2, \ldots, d, j=1,2, \ldots \\
B, C^{i}, D^{j}, G^{i}, V^{i}, K^{j}, L^{j}:[0, T] \times \Omega \longrightarrow R^{n \times m}, \\
i=1,2, \ldots, d, j=1,2, \ldots \\
E, W^{i}, M^{j}:[0, T] \times \Omega \longrightarrow R^{n \times k}, \\
i=1,2, \ldots, d, j=1,2, \ldots \\
P, Q^{i}, R^{j}, \widetilde{L}, \widetilde{M}^{i}, \widetilde{K}^{j}:[0, T] \times \Omega \longrightarrow R^{m \times m}, \\
i=1,2, \ldots, d, j=1,2, \ldots \\
N:[0, T] \times \Omega \longrightarrow R^{m \times n}, \\
S:[0, T] \times \Omega \longrightarrow R^{m \times k}, \\
\widetilde{N}:[0, T] \times \Omega \longrightarrow R^{k \times k} ;
\end{gathered}
$$

the $\mathscr{F}_{T}$-predictable random matrix $\widetilde{Q}: \Omega \rightarrow R^{n \times n}$ and the $\mathscr{F}_{0}$-predictable stochastic matrix $\widetilde{P}: \Omega \rightarrow R^{m \times m}$ are all 
uniformly bounded. And $a \in \mathbb{R}^{n \times n}, h \in L^{2}\left(\Omega, \mathscr{F}_{T}, P ; \mathbb{R}^{m \times n}\right)$, and $\xi \in L^{2}\left(\Omega, \mathscr{F}_{T}, P ; \mathbb{R}^{m}\right)$.

To study this problem, we need the assumptions on the coefficients as follows.

Assumption 11. The state weighting matrix processes $\widetilde{R}, \widetilde{L}, \widetilde{M}^{i}$, and $\widetilde{K}^{j}, i=1,2, \ldots, d, j=1,2, \ldots$, and the control weighting matrix process $\widetilde{N}$ and random matrixes $\widetilde{Q}$ and $\widetilde{P}$ are almost everywhere almost surely symmetric and nonnegative. Furthermore, $\widetilde{N}$ is almost everywhere almost surely uniformly positive; that is, $N \geq \delta I$, for some positive constant $\delta$ almost everywhere almost surely.

Assumption 12. For the control processes there is no further constraint:

$$
\begin{aligned}
& U_{a d}=\{u(\cdot): u(\cdot) \\
& \text { is } \mathscr{F}_{t} \text {-predictable with values in } \mathbb{R}^{k}, \\
& \left.E \int_{0}^{T}|u(t)| d t<\infty\right\} .
\end{aligned}
$$

If we denote the norm of $U_{a d}$ by

$$
\|u(\cdot)\|_{U_{a d}}=E \sqrt{\int_{0}^{T}|u(t)|^{2} d t}
$$

then $U_{a d}$ is a Hilbert space. And by Lemma 3 we also know that the linear FBSDEL (60) has a unique solution; that is, the linear quadratic problem is well defined.

Theorem 13. Under Assumptions 11 and 12, LQ problems (60) and (61) have a unique optimal control, and the optimal control is

$$
\begin{aligned}
u_{t} & =-\frac{1}{2} \widetilde{N}_{t}^{-1}\left[E_{t}^{T} q_{t}-S_{t}^{T} p_{t}+\sum_{i=1}^{d}\left(W_{t}^{i}\right)^{T} k_{t}^{i}\right. \\
& \left.+\sum_{i=1}^{\infty}\left(M_{t}^{i}\right)^{T} \rho_{t}^{i}\right]
\end{aligned}
$$

here $p, q, k$, and $\rho$ are the solution of the following adjoint FBSDEL:

$$
\begin{aligned}
d p_{t} & =\left[P_{t} p_{t}-B_{t}^{T} q_{t}-\sum_{i=1}^{d}\left(G_{t}^{i}\right)^{T} k_{t}^{i}-\sum_{i=1}^{\infty}\left(K_{t}^{i}\right)^{T} \rho_{t}^{i}\right. \\
& \left.-2 \widetilde{L}_{t} y_{t}\right] d t \\
& -\sum_{i=1}^{d}\left[Q_{t}^{i} p_{t}-\left(C_{t}^{i}\right)^{T} q_{t}-\left(V_{t}^{i}\right)^{T} k_{t}^{i}-2\left(\widetilde{M}_{t}^{i}\right) z_{t}^{i}\right] d B_{t}^{i}
\end{aligned}
$$

$$
\begin{aligned}
& -\sum_{i=1}^{\infty}\left[R_{t}^{i} p_{t}^{i}-\left(D_{t}^{i}\right)^{T} q_{t}-\left(L_{t}^{i}\right)^{T} \rho_{t}-2 \widetilde{K}_{t}^{i} r_{t}^{i}\right] d H_{t}^{i}, \\
& -d q_{t}=\left[N_{t}^{T} p_{t}-A_{t} q_{t}-\sum_{i=1}^{d} F_{t}^{i} k_{t}^{i}-\sum_{i=1}^{\infty} J_{t}^{\mathrm{i}} \rho_{t}^{i}-2 \widetilde{R}_{t} x_{t}\right] d t \\
& \quad-\sum_{i=1}^{d} k_{t}^{i} d B_{t}-\sum_{i=1}^{\infty} \rho_{t}^{i} d H_{t}^{i}, \\
& p_{0}=-2 \widetilde{P} y_{0}, \\
& q_{T}=2 \widetilde{Q} x_{T}-h p_{T} .
\end{aligned}
$$

Proof. From Assumptions 11 and 12 and inequality (29), we can verify that the cost function $J(u(\cdot))(61)$ is strictly convex and continuous over $U_{a d}$ and

$$
\lim _{\|u(\cdot)\|_{U_{a d}} \rightarrow \infty} J(u(\cdot))=\infty
$$

then, from Lemma 5.3 in [18], the cost function has a unique minimal value and, together with Lemma 3 (existence and uniqueness theorem of FBSDEL), the LQ problem has a unique optimal control. Next, we will prove that the optimal control $u_{t}$ has an expression as (65).

Let $\left(x_{t}, y_{t}, z_{t}, r_{t}\right)$ be the optimal state process corresponding to the optimal control $u_{t}$ and let $\left(p_{t}, q_{t}, k_{t}, \rho_{t}\right)$ be the unique solution to adjoint equation (66) corresponding to the optimal pair $\left(u_{t} ; x_{t}, y_{t}, z_{t}, r_{t}\right)$; then the Hamiltonian function

$$
\begin{aligned}
& H(t, x, y, z, r, u, p, q, k, \rho)=-\langle p, \\
& \left.\left(N_{t} x_{t}+P_{t} y_{t}+\sum_{i=1}^{d} Q_{t}^{i} z_{t}^{i}+\sum_{i=1}^{\infty} R_{t}^{i} r_{t}^{i}+S_{t} u_{t}\right)\right\rangle+\langle q, \\
& \left.\left(A_{t} x_{t}+B_{t} y_{t}+\sum_{i=1}^{d} C_{t}^{i} z_{t}^{i}+\sum_{i=1}^{\infty} D_{t}^{i} r_{t}^{i}+E_{t} u_{t}\right)\right\rangle+\langle k, \\
& \left.\sum_{i=1}^{d}\left(F_{t}^{i} x_{t}+G_{t}^{i} y_{t}+V_{t}^{i} z_{t}^{i}+W_{t}^{i} u_{t}\right)\right\rangle+\langle\rho, \\
& \left.\sum_{i=1}^{\infty}\left(J_{t}^{i} x_{t_{-}}+K_{t}^{i} y_{t_{-}}+L_{t}^{i} r_{t}^{i}+M_{t}^{i} u_{t}\right)\right\rangle+\left\langle\widetilde{R}_{t} x_{t}, x_{t}\right\rangle \\
& +\left\langle\widetilde{N}_{t} u_{t}, u_{t}\right\rangle+\left\langle\widetilde{L}_{t} y_{t}, y_{t}\right\rangle+\sum_{i=1}^{d}\left\langle\widetilde{M}_{t}^{i} z_{t}^{i}, z_{t}^{i}\right\rangle \\
& +\sum_{i=1}^{\infty}\left\langle\widetilde{K}_{t}^{i} r_{t}^{i}, r_{t}^{i}\right\rangle .
\end{aligned}
$$


From Theorem 9 and Assumption 12 we have

$$
\begin{aligned}
H_{u}= & -S_{t}^{T} p_{t}+E_{t}^{T} q_{t}+\sum_{i=1}^{d}\left(W_{t}^{i}\right)^{T} k_{t}^{i}+\sum_{i=1}^{\infty}\left(M_{t}^{i}\right)^{T} \rho_{t}^{i} \\
& +2 N_{t} u_{t}=0
\end{aligned}
$$

that is

$$
\begin{aligned}
u_{t} & =-\frac{1}{2} \widetilde{N}_{t}^{-1}\left[E_{t}^{T} q_{t}-S_{t}^{T} p_{t}+\sum_{i=1}^{d}\left(W_{t}^{i}\right)^{T} k_{t}^{i}\right. \\
& \left.+\sum_{i=1}^{\infty}\left(M_{t}^{i}\right)^{T} \rho_{t}^{i}\right] ;
\end{aligned}
$$

then (65) holds.

The proof is completed.

\section{Conclusion}

In this paper, the continuity result depending on parameters of forward-backward stochastic differential equation driven by Lévy process is proved. Based on this result, we get the stochastic maximum principle for fully coupled forwardbackward stochastic control system driven by Lévy process. And then we use the stochastic maximum principle to solve LQ problem.

\section{Conflict of Interests}

The authors declare that there is no conflict of interests regarding the publication of this paper.

\section{Acknowledgments}

This work was supported by the National Natural Science Foundation of China (no. 11271007), the SDUET Research Fund (no. 2012kytd105), and the Doctoral Fund of the Ministry of Education of China (no. 20123718110010).

\section{References}

[1] S. Peng and Z. Wu, "Fully coupled forward-backward stochastic differential equations and applications to optimal control," SIAM Journal on Control and Optimization, vol. 37, no. 3, pp. 825-843, 1999.

[2] S. Peng, "Backward stochastic differential equations and applications to optimal control," Applied Mathematics and Optimization, vol. 27, no. 2, pp. 125-144, 1993.

[3] Z. Wu, "Maximum principle for optimal control problem of fully coupled forward-backward stochastic systems," Systems Science and Mathematical Sciences, vol. 11, no. 3, pp. 249-259, 1998.

[4] J.-T. Shi and Z. Wu, "The maximum principle for fully coupled forward-backward stochastic control system," Acta Automatica Sinica, vol. 32, no. 2, pp. 161-169, 2006.

[5] Z. Wu, "Forward-backward stochastic differential equations with Brownian motion and Poisson process," Acta Mathematicae Applicatae Sinica, vol. 15, no. 4, pp. 433-443, 1999.
[6] J. Shi and Z. Wu, "Maximum principle for fully coupled forward-backward stochastic control system with random jumps," in Proceedings of the 26th Chinese Control Conference (CCC '07), pp. 375-380, Hunan, China, July 2007.

[7] J. Shi and Z. Wu, "Maximum principle for forward-backward stochastic control system with random jumps and applications to finance," Journal of Systems Science and Complexity, vol. 23, no. 2, pp. 219-231, 2010.

[8] J. Shi and Z. Wu, "Necessary condition for optimal control of fully coupled forward-backward stochastic system with random jumps," in Proceedings of the 31st Chinese Control Conference (CCC '12), pp. 1620-1628, Hefei, China, July 2012.

[9] J. Shi, "Necessary conditions for optimal control of forwardbackward stochastic systems with random jumps," International Journal of Stochastic Analysis, vol. 2012, Article ID 258674, 50 pages, 2012.

[10] F. Baghery, N. Khelfallah, B. Mezerdi, and I. Turpin, "Fully coupled forward backward stochastic dfferential wquations driven by Lévy processes and application to dfferential games," Random Operators and Stochastic Equations, vol. 22, no. 3, pp. 151-161, 2014.

[11] Q. Zhu, "Asymptotic stability in the pth moment for stochastic differential equations with Lévy noise," Journal of Mathematical Analysis and Applications, vol. 416, no. 1, pp. 126-142, 2014.

[12] D. Nualart and D. Schoutens, "Chaotic and predicatable respresentation for Lévy processes with applications in finance," Journal of Systems Science and Complexity, vol. 90, pp. 109-122, 2000.

[13] D. Nualart and D. Schoutens, "Backward stochastic differential equations and Feynman-Kac formula for Lévy processes, with applications in finance," Bernoulli, vol. 7, pp. 761-776, 2001.

[14] K. Bahlali, M. Eddahbi, and E. Essaky, "BSDE associated with Lévy processes and application to PDIE," Journal of Applied Mathematics and Stochastic Analysis, vol. 16, no. 1, pp. 1-17, 2003.

[15] K.-I. Mitsui and Y. Tabata, "A stochastic linear-quadratic problem with Lévy processes and its application to finance," Stochastic Processes and Their Applications, vol. 118, no. 1, pp. 120-152, 2008.

[16] H. Tang and Z. Wu, "Stochastic dfferential equations and stochastic linear quadratic optimal control problem with Lévy processes," Journal of Systems Science \& Complexity, vol. 22, no. 1, pp. 122-136, 2009.

[17] Q. Meng and M. Tang, "Necessary and sufficient conditions for optimal control of stochastic systems associated with Lévy processes," Science in China: Series F: Information Sciences, vol. 52, no. 11, pp. 1982-1992, 2009.

[18] M. Tang and Q. Zhang, "Optimal variational principle for backward stochastic control systems associated with Lévy processes," Science China Mathematics, vol. 55, no. 4, pp. 745-761, 2012.

[19] J. Z. Lin, "A general stochastic maximum principle for optimal control of stochastic systems driven by multidimensional Teugel's martingales," http://arxiv.org/abs/1205.6315vl.

[20] F. Zhang, M. N. Tang, and Q. X. Meng, "Sochastic maximum principle for forward-backward stochastic control systems associated with Lévy processes," Chinese Annals of Mathematics, vol. 35, no. 1, pp. 83-100, 2014 (Chinese).

[21] P. Protter, Stochastic Integration and Differential Equations, vol. 21 of Applications of Mathematics, Springer, Berlin, Germany, 1990. 


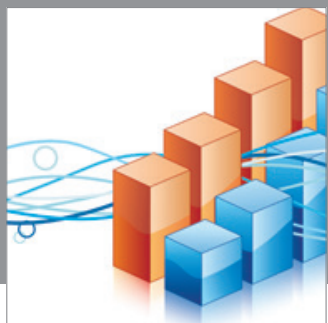

Advances in

Operations Research

mansans

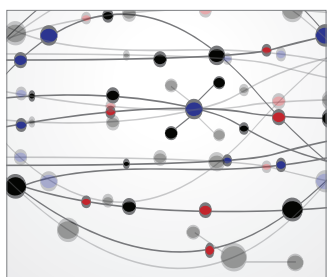

The Scientific World Journal
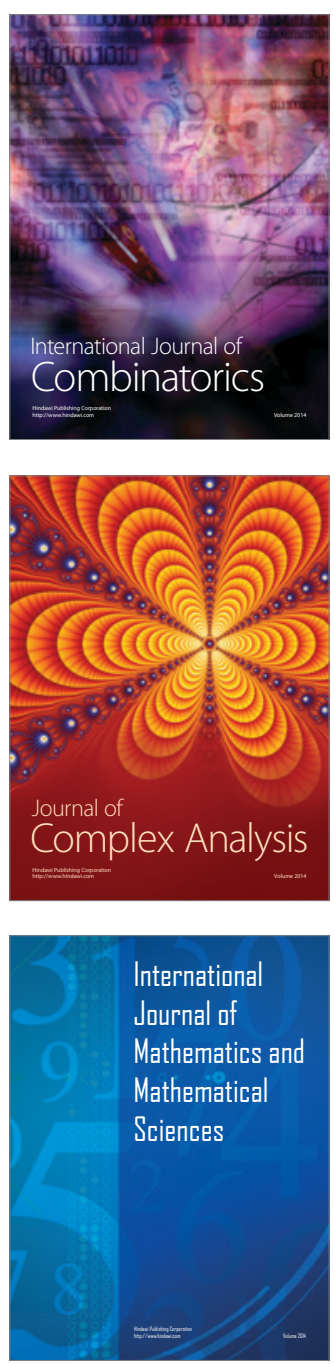
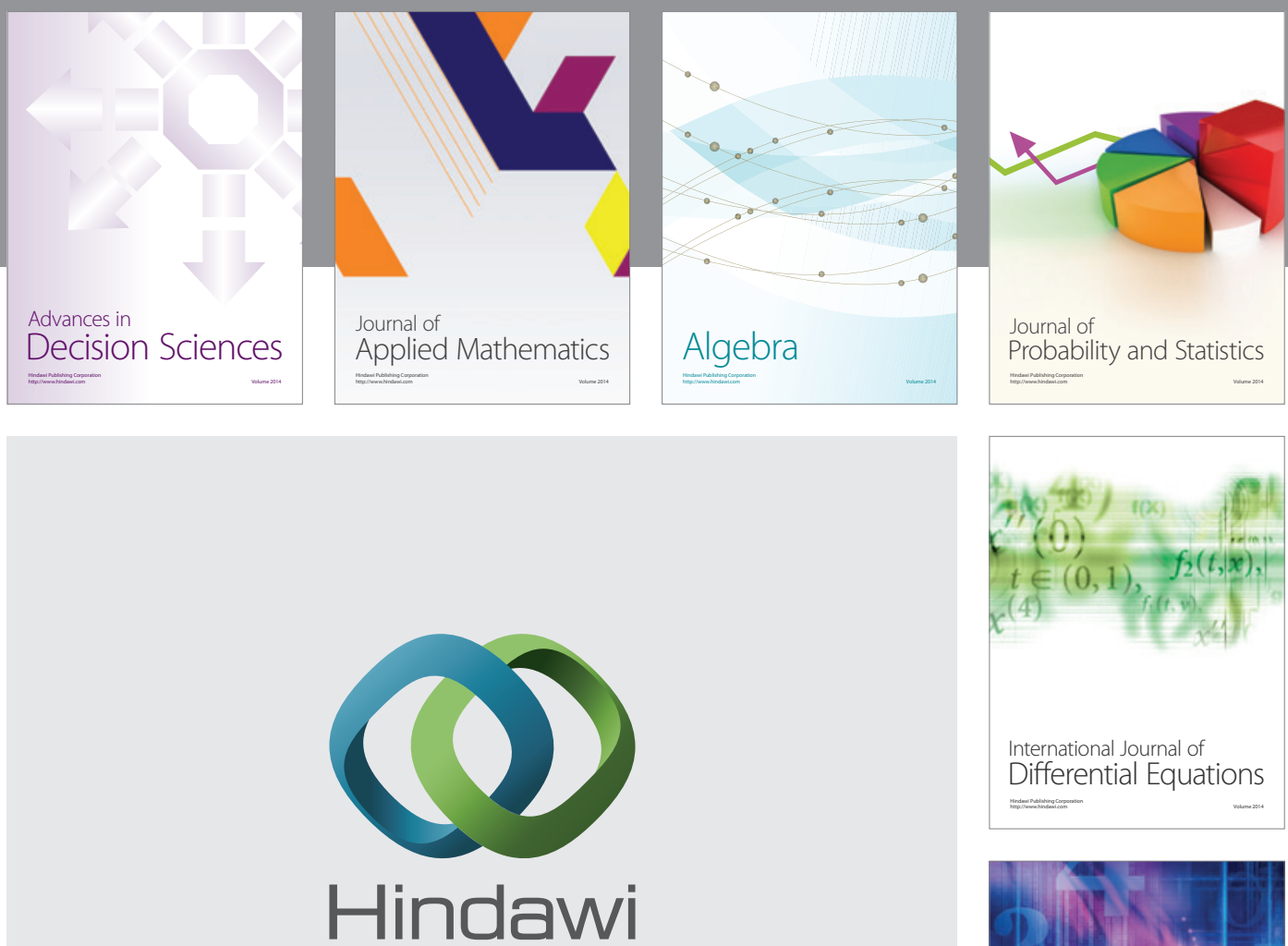

Submit your manuscripts at http://www.hindawi.com
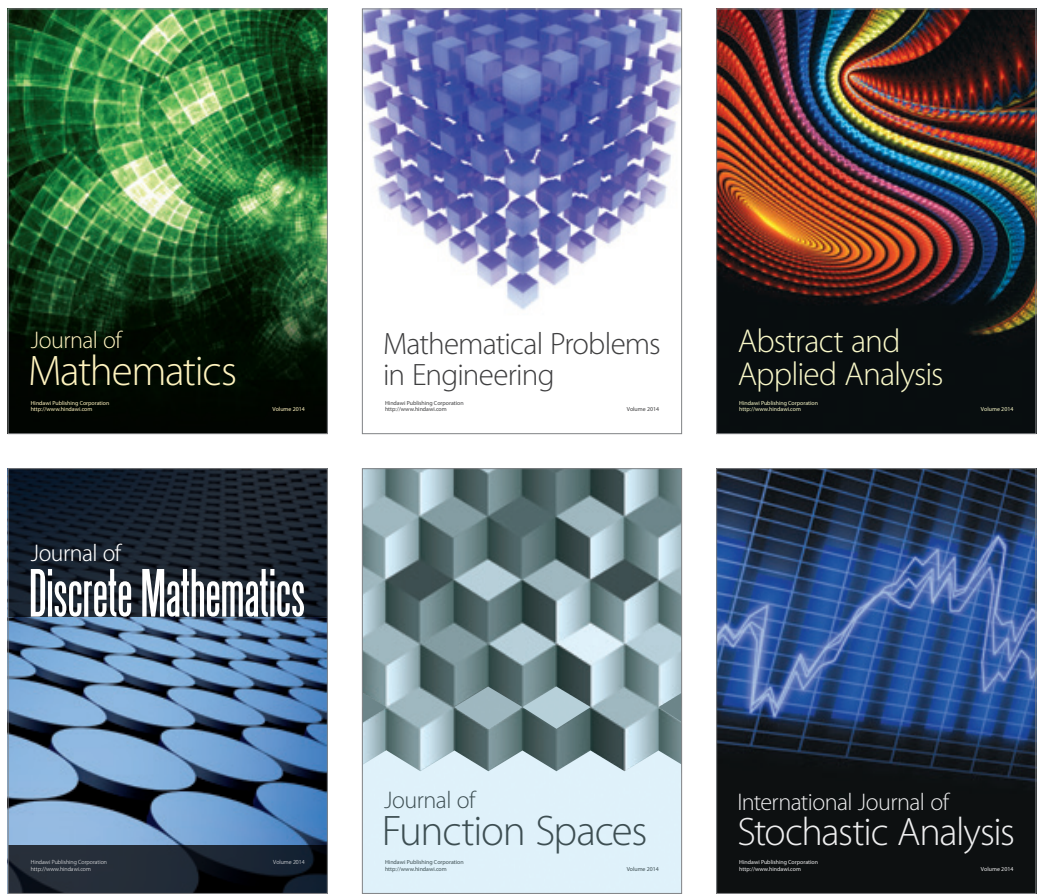

Journal of

Function Spaces

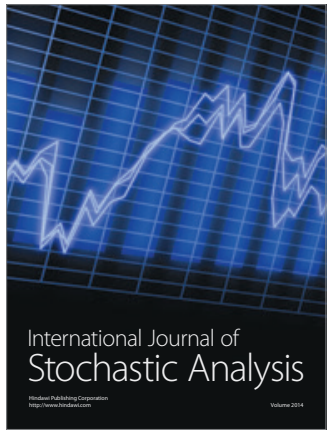

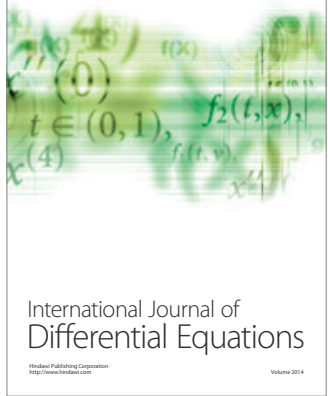
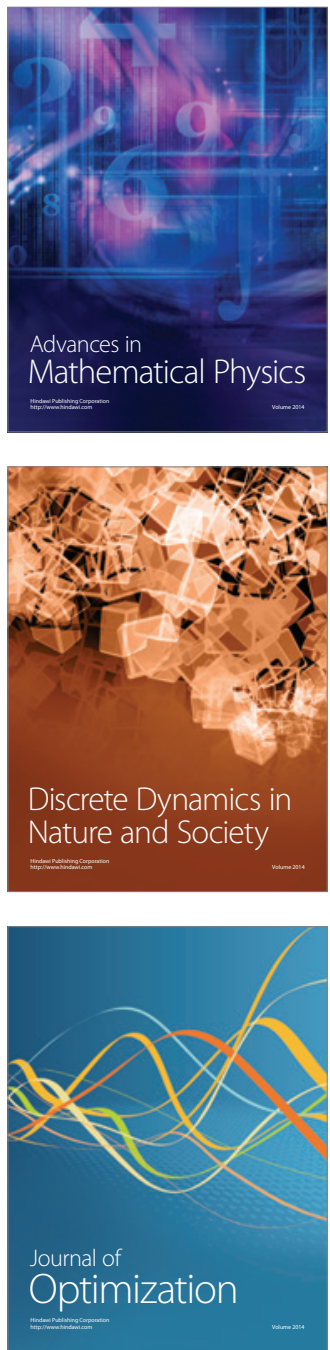